\title{
Upregulation of ADAMTS-7 and downregulation of COMP are associated with spontaneous abortion
}

\author{
YANG MU, DAN-NI ZHOU, NA-NA YAN, JIN-LI DING and JING YANG \\ Reproductive Medicine Center, Renmin Hospital of Wuhan University, \\ Hubei Clinic Research Center for Assisted Reproductive Technology and Embryonic Development, \\ Wuhan University, Wuhan, Hubei 430060, P.R. China
}

Received February 13, 2018; Accepted August 7, 2018

DOI: $10.3892 / \mathrm{mmr} .2019 .9898$

\begin{abstract}
A disintegrin and metalloproteinase with thrombospondin motifs 7 (ADAMTS-7) has been revealed to serve an important role in inflammation-associated diseases. However, the role of ADAMTS-7 in spontaneous abortion (SA) remains unclear. In the present study, human and mouse decidual tissues were used to detect the expression of ADAMTS-7 and cartilage oligomeric matrix protein (COMP) in mice with lipopolysaccharide (LPS)-induced abortion (10 mice/group), and in SA humans and the corresponding control group (21 participants in the SA group and 15 participants in the control group). The results revealed that ADAMTS-7 expression was upregulated and that COMP expression was downregulated in the mouse decidual tissue of the LPS-induced abortion group, when compared with that of the normal control group. The results were further confirmed by western blot analysis and reverse transcription-quantitative polymerase chain reaction (RT-qPCR) analysis, which revealed increased ADAMTS-7 and decreased COMP expression at the protein and mRNA levels in mice treated with LPS. Additionally, the expression of ADAMTS-7 was negatively correlated with the expression of COMP in mice, with a correlation coefficient of $-0.936(\mathrm{P}<0.001)$. In addition, the expression of ADAMTS-7 and COMP exhibited was similar in the decidual tissue of SA patients when compared with the levels observed in the tissues of the normal control participants, as demonstrated
\end{abstract}

Correspondence to: Dr Jing Yang, Reproductive Medicine Center, Renmin Hospital of Wuhan University, Hubei Clinic Research Center for Assisted Reproductive Technology and Embryonic Development, Wuhan University, 238 Jiefang Road, Wuhan, Hubei 430060, P.R. China

E-mail: 13507182023@163.com

Abbreviations: SA, spontaneous abortion; COMP, cartilage oligomeric matrix protein; LPS, lipopolysaccharide; TNF- $\alpha$, tumor necrosis factor $\alpha$

Key words: A disintegrin and metalloproteinase with thrombospondin motifs 7, COMP, spontaneous abortion by increased ADAMTS-7 expression and decreased COMP expression. Western blotting and RT-qPCR analysis revealed that ADAMTS-7 was increased and COMP was decreased in the decidual tissue of SA subjects. The correlation analysis of ADAMTS-7 and COMP in human decidual tissue also revealed a similar result, with a correlation coefficient of -0.836 $(\mathrm{P}<0.001)$. The results of the present study demonstrated that ADAMTS-7 was upregulated and COMP was downregulated in the decidual tissues of humans and mice with SA, and a negative correlation was identified between the expression levels of ADAMTS-7 and COMP, thereby providing novel evidence for a better understanding of the pathogenesis of SA, which may lead to improvements in the clinical pregnancy outcomes of these individuals.

\section{Introduction}

Recurrent miscarriage is a highly heterogeneous disease, which is defined as at least three pregnancy losses before 22 weeks of gestation, and affects approximately $0.5-3 \%$ of fertile couples $(1,2)$. The pathogenesis of recurrent spontaneous miscarriage is complicated and remains mostly unknown. Immunological factors and inflammation have been reported to participate in the pathological process of recurrent miscarriage (3-9).

A disintegrin and metalloproteinase with thrombospondin motifs 7 (ADAMTS-7) is a proteolytic member of the ADAMTS family (a disintegrin and metalloproteinase with thrombospondin-like motifs) and has been identified to exert important effects on inflammatory diseases, such as arthritis and atherosclerosis (10-12). Previous studies have demonstrated that systematic inflammation is correlated with recurrent implantation failure and recurrent spontaneous abortion (SA) in humans (13). Over the past few decades, Th17 cells have been reported to be involved in women with reproductive failure. As a secreted factor of Th17 cells, the expression of IL-17 in decidua is clearly higher in inevitable abortion and in unexplained recurrent SA individuals than in the control group $(14,15)$. In addition, IL-17A, an important regulatory factor participating in the pathological process of recurrent SA, has been found to enhance the expression of ADAMTS-7 in human nucleus pulposus cells (16). Therefore, a potential role of ADAMTS-7 in SA was proposed. 
Accumulative evidence has indicated that women with adverse pregnancy outcomes are associated with a higher risk of suffering from cardiovascular disease, with preeclampsia as the most documented $(17,18)$. It is also reported that women with a history of recurrent miscarriage are more likely to experience cardiovascular disease (19-21). Therefore, an underlying association was proposed between the molecular mechanism of recurrent miscarriage and cardiovascular disease. A recent study found that circulating ADAMTS-7 levels could reflect the degree of ventricle remodeling after acute myocardial infarction in a rat model (22). Moreover, it was also reported that ADMATS-7 participates in the pathological process of atherosclerosis through degradation of cartilage oligomeric matrix protein (COMP) (23-25), which has been confirmed to be involved in some pathophysiological pathways, including vascular calcification, atherosclerosis and neointima formation post-injury $(26,27)$.

Our study aimed to detect the expression of ADMATS-7 and its substrate COMP in the decidua of both LPS-treated mice and humans with a history of SA as well as the corresponding control group. We aimed to find a potential association between the expression of ADAMTS-7 and COMP in the decidua of LPS-treated mice and SA humans, which would be helpful to further understand the pathogenesis of recurrent SA in order to find effective measures for treating this disease.

\section{Materials and methods}

Animals. Eight- to ten-week-old C57/BL mice were purchased from Beijing Vital River Laboratory Animal Technology Co., Ltd. (Beijing, China). The mice were housed in an environment controlled for temperature $\left(22 \sim 24^{\circ} \mathrm{C}\right)$ and conditions of light (12 $\mathrm{h}$ light and $12 \mathrm{~h}$ darkness), with free access to standard mouse food and water. 2 female mice were caged with one male mouse every other day, and vaginal plugs were checked the next morning as a sign of mating behavior. The day of plug detection was defined as embryonic day 0.5 (E 0.5). The pregnant mice were randomly divided into lipopolysaccharide (LPS) group and control group, with 10 mice in each group. LPS (Sigma, Saint Louis, MO, USA) at a dose of $2.5 \mu \mathrm{g}$ dissolved in saline was intraperitoneally injected in pregnant C57BL/6 female mice at E 7.5 to induce abortion. Mice in control group received the same volume of saline as the vehicle. At E10.5, the mice were sacrificed and the embryo resorption rate were calculated by dividing the number of resorbed embryos by the number of implantations, and the decidua were collected for further experiments. All of the experiments involving animals in this study were carried out in accordance with the protocols of the Guidelines for the Care and Use of Laboratory Animals published by the United States National Institutes of Health (NIH Publication, revised 2011) and the Guidelines for the Care and Use of Laboratory Animals of the Chinese Animal Welfare Committee. The procedures were approved by the Animal Use Committees of Renmin Hospital of Wuhan University (Wuhan, China).

Human study. The procedures involving human experiments were performed in accordance with the Declaration of Helsinki and were approved by the Human Research
Ethics Committees of Renmin Hospital of Wuhan University (Wuhan, China). All participants were patients who went to the department of obstetrics and gynecology for treatment, and informed consent was signed by each participant. People with a history of SA before 22 weeks of gestation were divided into the SA group. People who went to the hospital for artificial abortion were placed into the control group. Subjects who had a history of autoimmune- or thyroid-related disease, uterine malformation, ultrasonographic evidence of hydrosalpinx, and hormone therapy within three months were excluded from this study. All participants shared similar baseline demographics. Finally, a total of 36 people were involved in this study, with 15 people in the control group and 21 people in the SA group.

Immunohistochemistry. Decidua tissue was collected and washed by PBS three times. The decidua tissues were then put into $10 \%$ formaldehyde at room temperature for $24 \mathrm{~h}$, followed by deparaffinization and dehydration. Then, the samples were cut into 5- $\mu \mathrm{m}$ sections for further immunohistochemistry detection. Endogenous peroxidase was blocked with $3 \% \mathrm{H}_{2} \mathrm{O}_{2}$. Non-specific staining was blocked using $5 \%$ bovine serum albumin for $20 \mathrm{~min}$. Subsequently, the sections were incubated with primary antibody targeted to ADAMTS-7 (1:200) and COMP (1:200) at $4^{\circ} \mathrm{C}$ overnight. Thereafter, the decidua sections were exposed to biotinylated sheep anti-rabbit immunoglobulin $\mathrm{G}$ solution (Proteintech Group, Inc.) at $37^{\circ} \mathrm{C}$ for $30 \mathrm{~min}$ followed by incubation with horseradish peroxidase-labeled streptavidin (Proteintech Group, Inc.) at $37^{\circ} \mathrm{C}$ for $30 \mathrm{~min}$. After washing with PBS three times, the sections were counterstained with hematoxylin and dehydrated. Then, the sections were observed under a microscope (Nikon E100; Nikon Corporation, Tokyo, Japan), and the photomicrographs were obtained by Photo Imaging System (Canon 600D; Canon, Inc., Tokyo, Japan). A brown color was regarded as a positive signal. The optic density was detected by Image-Pro Plus 6.0, and the mean of the integrated optical density was obtained to represent the expression of ADAMTS-7 and COMP.

Western blot and reverse transcription-quantitative polymerase chain reaction ( $R T-q P C R)$. Briefly, the decidua were weighed, cut and homogenized in a 1:10 (w/v) RIPA buffer using a homogenizer. After chilling on ice for $30 \mathrm{~min}$, the suspension was centrifuged at $1,409 \mathrm{x} \mathrm{g}$ at $4^{\circ} \mathrm{C}$ for $15 \mathrm{~min}$ and the supernatants were collected. Then, the protein concentration was determined using a BCA Protein Assay kit (Pierce; Thermo Fisher Scientific, Inc., Waltham, MA, USA). A total of $20 \mu \mathrm{g}$ protein were separated on SDS-PAGE gels and then transferred to a PVDF membrane. Subsequently, the membrane was blocked with $5 \%$ non-fat milk dissolved in TBST at room temperature for $2 \mathrm{~h}$. Then, the membrane was incubated with the following primary antibodies at $4^{\circ} \mathrm{C}$ overnight: Rabbit anti-GAPDH antibody $(1: 5,000)$, rabbit anti-ADAMTS-7 antibody (1:1,000, ab28557; Abcam, Cambridge, UK) and rabbit anti-COMP antibody $(1: 1,000$, ab42225; Abcam). After washing the membrane with TBST three times, the membranes were incubated with secondary antibody for $2 \mathrm{~h}$ at room temperature. Finally, the bands were scanned using a two-color infrared imaging system (Odyssey; LI-COR, Lincoln, NE, USA). Band intensity was quantified 
using Odyssey, as described previously, and the densities of the bands were normalized to GAPDH.

Total RNA was extracted using the TRIzol (Invitrogen; Thermo Fisher Scientific, Inc.) method according to the manufacturer's instructions. RNA concentration was detected using an absorbance at 260 and $280 \mathrm{~nm}$ (A260/280). Total RNA was reverse-transcribed into cDNAs using a PrimeScript ${ }^{\mathrm{TM}}$ RT-PCR Kit (04896866001; Roche Diagnostics, Indianapolis, IN, USA). Quantitative RT-PCR analysis was conducted using LightCycler 480 SYBR Green 1 Master Mix (04707516001; Roche Diagnostics). The primer sequences for Adamts-7 were as follows: Forward: 5'-TCACCAGGTTCCTTGACCGTG-3' and reverse: 5'-CCAGCTTGGAGTGACAGGTGGT-3'. The primer sequences for Comp were as follows: Forward: 5'-GCGCCA GTGTCGCAAGGACAA-3' and reverse: 5'-TGGGTTTCG AACCAGCGGGC-3'. The primer sequences for Gapdh were as follows: Forward: 5'-TCATCAACGGGAAGCCCATC-3' and reverse: 5'-CTCGTGGTTCACACCCATCA-3'. Expression of Adamts-7 and Comp was determined using the $2^{-\Delta \Delta \mathrm{Cq}}$ method and normalized to Gapdh (28).

Statistical analysis. All data were expressed as the mean \pm standard deviation. The group sizes of the experiments were estimated based on power analysis of ADAMTS-7 mRNA expression, with an $\alpha$ error of $5 \%$ and a power of $80 \%$, which is consistent with a published article (29). To detect a $10 \%$ change in ADAMTS-7 mRNA expression with an expected SD of $5 \%$, we needed 5 animals per group. We had 10 mice in each group, and for humans, the sample size in the SA and control groups was 21 and 15, respectively, which fulfilled the requirement. Statistical analysis was performed with SPSS 22.0 (IBM Corp., Armonk, NY, USA). All the data in the present study were normally distributed $(\mathrm{P}<0.05)$, as determined by the Kolmogorov-Smirnov's test. The difference between two groups was then evaluated using independent samples t-tests (two-sided). The correlation between ADMATS-7 and COMP was assessed by Spearman's analysis. $\mathrm{P}<0.05$ was considered to indicate a statistically significant difference.

\section{Results}

The expression of ADAMTS-7 and COMP in mouse decidua of the LPS-induced abortion group and the control group. Embryo resorption was $100 \%$ after LPS treatment. As shown in Fig. 1, the expression of ADAMTS-7 was clearly increased in the mouse decidua of the LPS-induced abortion group (Fig. 1). As a downstream substrate of ADAMTS-7, the expression of COMP was clearly decreased in the mouse decidua of LPS group compared with that of the control group (Fig. 1). Spearman's analysis showed that the expression of ADMATS-7 was negatively correlated with the expression of COMP in mouse decidual tissue, which had a correlation coefficient of $-0.936(\mathrm{P}<0.001$; Fig. 2).

Western blot analysis revealed that the protein level of ADAMTS-7 was increased in the LPS group compared with the control group. Additionally, the expression of COMP was decreased in the decidual tissue of the LPS group (Fig. 3A). The mRNA levels of Adamts-7 and Comp showed similar results, as demonstrated by an increased Adamts -7 mRNA level and decreased Comp mRNA level (Fig. 3B).
A
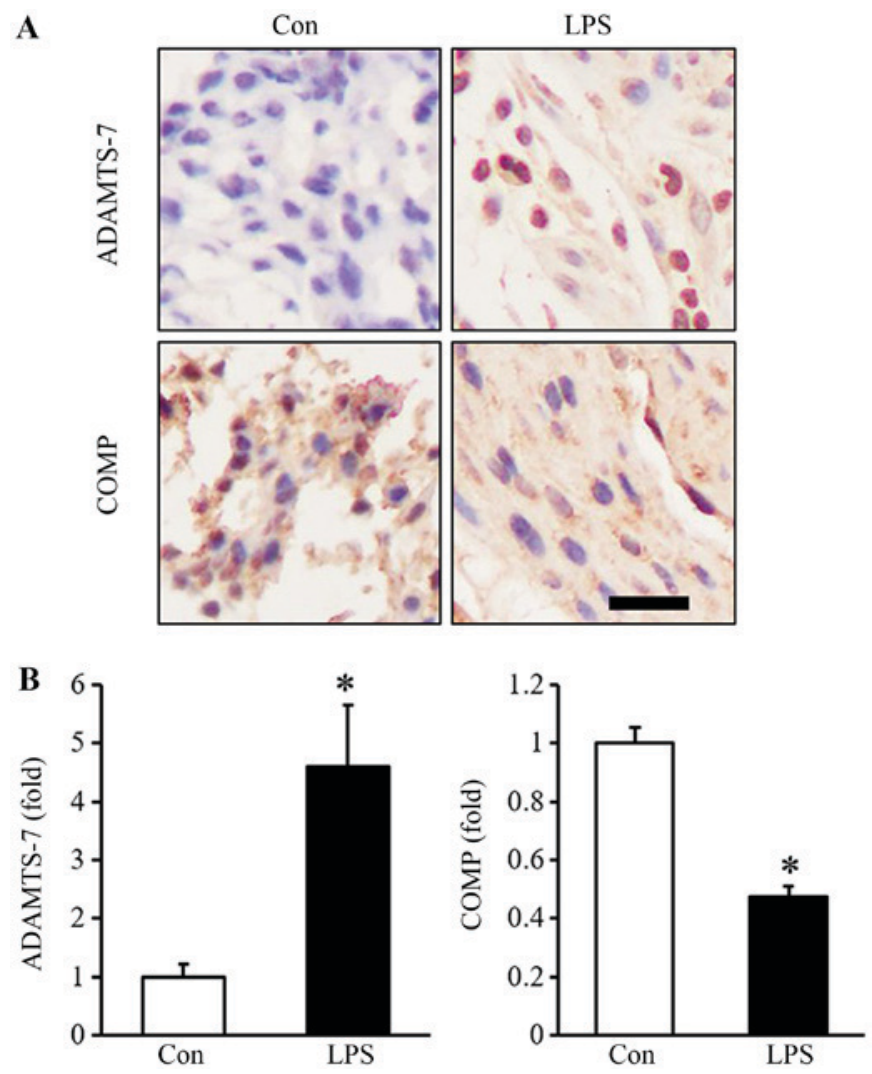

Figure 1. Expression of ADAMTS-7 and COMP in mouse decidua by immunohistological analysis. (A) Immunostaining of ADAMTS-7 and COMP in the mouse decidua of the control and LPS-induced abortion group $(\mathrm{n}=6)$. Scale bar, $50 \mu \mathrm{m}$; magnification, $\mathrm{x} 400$. (B) Mean optical density of ADAMTS-7 and COMP in the mouse decidual tissue of the control and LPS-induced abortion group $(n=6)$. ${ }^{*} \mathrm{P}<0.05$ vs. Con. ADAMTS-7, A disintegrin and metalloproteinase with thrombospondin motifs 7; COMP, cartilage oligomeric matrix protein; Con, control; LPS, lipopolysaccharide.

The expression of ADAMTS-7 and COMP in the human decidua of the SA group and the control group. As shown in Fig. 4, the expression of ADAMTS-7 was clearly increased in the human decidual tissue of the SA group. The expression of COMP was decreased in the human decidua of the SA group compared with that of the control group. Spearman's analysis showed that the expression of ADMATS-7 was negatively correlated with the expression of COMP in human decidual tissue, which had a correlation coefficient of -0.836 ( $\mathrm{P}<0.001$; Fig. 5).

The protein levels of ADAMTS-7 and COMP showed similar results the mice model, manifested as increased ADMATS-7 and decreased COMP levels (Fig. 6A). The mRNA level of Adamts-7 was increased, and the mRNA level of COMP was decreased in the SA group compared with the control group (Fig. 6B).

\section{Discussion}

In the present study, we first detected the expression of ADAMTS-7 and COMP in the decidual tissues of both LPS-treated mice and human subjects with SA. As demonstrated, ADAMTS-7 was increased, and COMP was decreased in the decidual tissue of the LPS-treated mice model compared with that of the control group, as depicted by immunohistochemistry, western blot and RT-qPCR. Moreover, we also revealed 


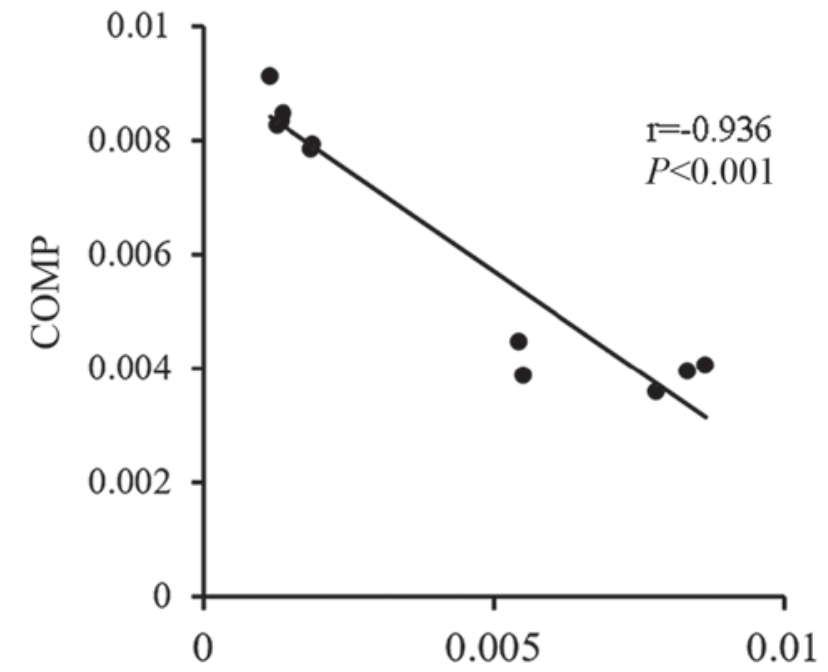

ADAMTS-7

Figure 2. Spearman's analysis of ADAMTS-7 and COMP in mouse decidua. ADAMTS-7, A disintegrin and metalloproteinase with thrombospondin motifs 7; COMP, cartilage oligomeric matrix protein.

A
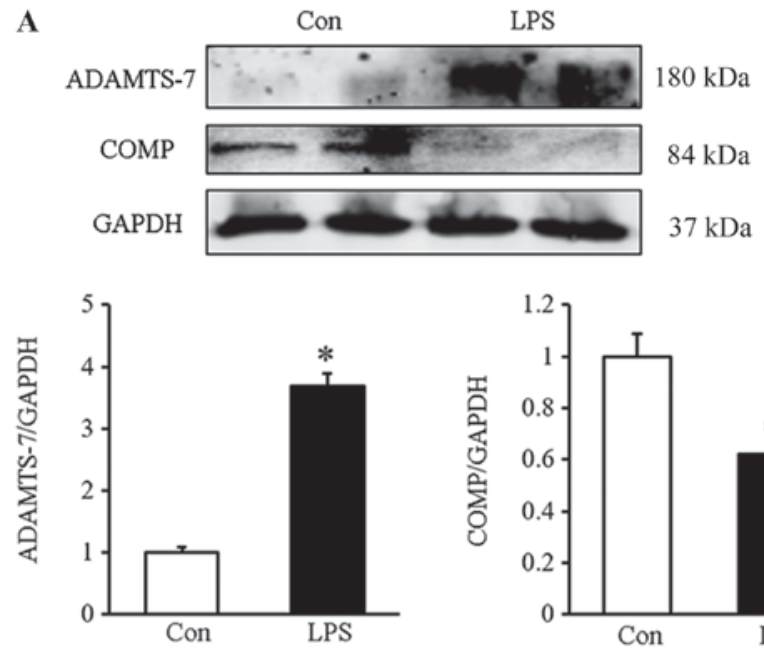

B
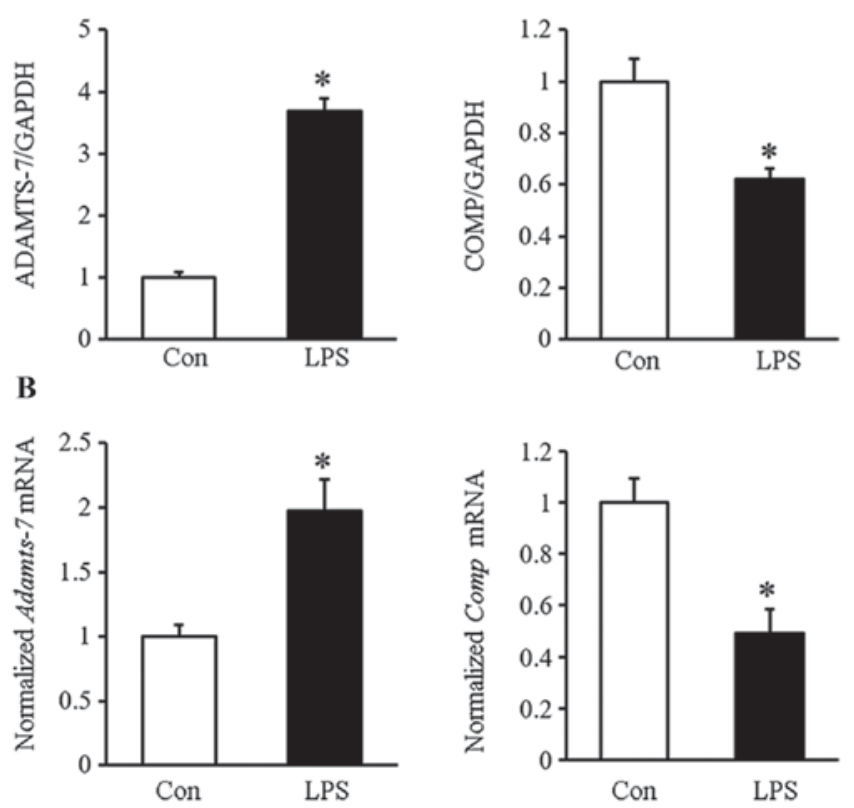

Figure 3. Expression of ADAMTS-7 and COMP in mouse decidua. (A) Protein levels of ADAMTS-7 and COMP in mouse decidua by western blotting (n=5). (B) mRNA level of Adamts-7 and Comp in mouse decidua by reverse transcription-quantitative polymerase chain reaction $(n=5)$. ${ }^{*} \mathrm{P}<0.05$ vs. Con. ADAMTS-7, A disintegrin and metalloproteinase with thrombospondin motifs 7; COMP, cartilage oligomeric matrix protein; Con, control; LPS, lipopolysaccharide.

that ADAMTS-7 was negatively correlated with COMP in the decidual tissue of mice. The results in humans were similar to the results in mice. ADAMTS-7 was upregulated, and COMP
A
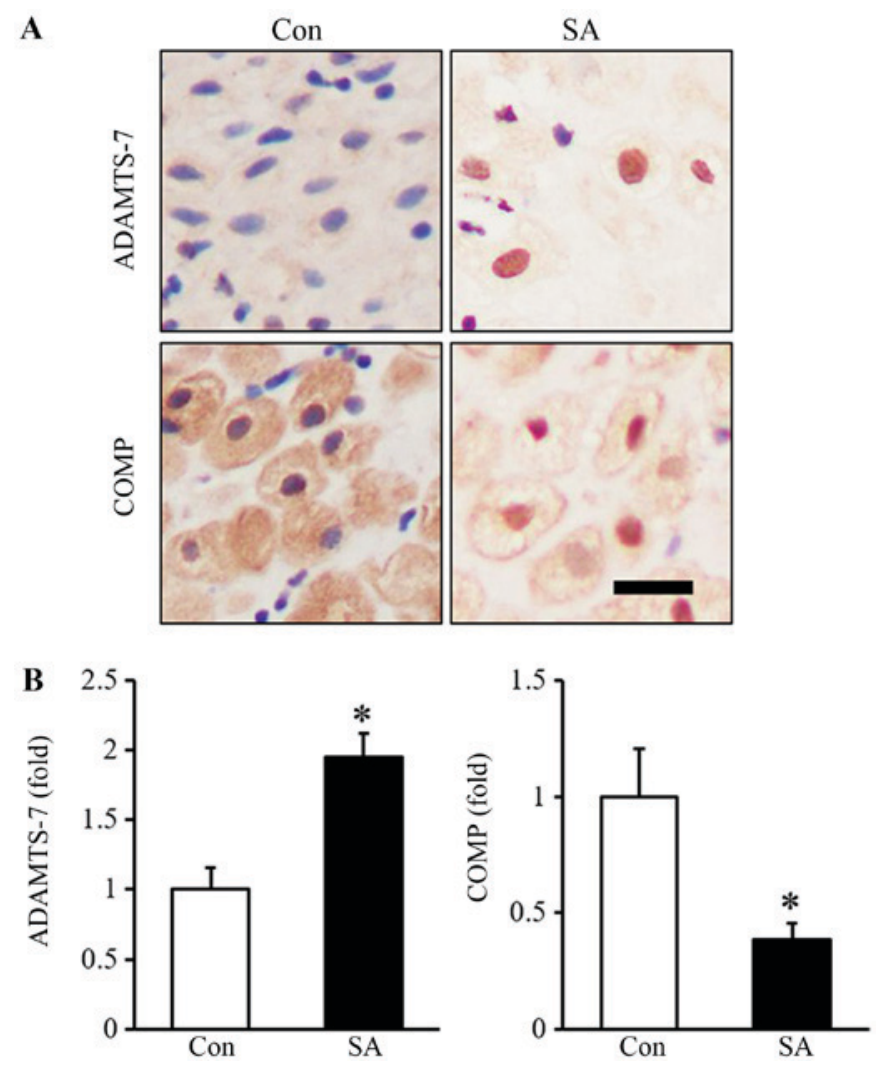

Figure 4. Expression of ADAMTS-7 and COMP in human decidua by immunohistological analysis. (A) Immunostaining of ADAMTS-7 and COMP on human decidua of the control $(\mathrm{n}=15)$ and SA groups $(\mathrm{n}=21)$. Scale bar, $50 \mu \mathrm{m}$; magnification, $x 400$. (B) Mean optical density of ADAMTS-7 and COMP in the human decidual tissue of the control $(n=15)$ and SA groups $(n=21)$. ${ }^{*} \mathrm{P}<0.05$ vs. Con. ADAMTS-7, A disintegrin and metalloproteinase with thrombospondin motifs 7; COMP, cartilage oligomeric matrix protein; Con, control; SA, spontaneous abortion.

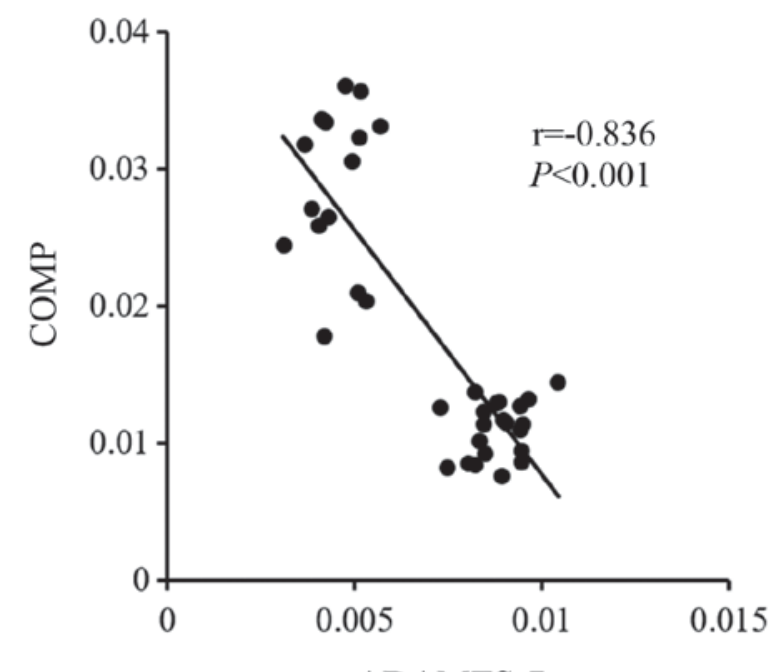

ADAMTS-7

Figure 5. Spearman's analysis of ADAMTS-7 and COMP in human decidua. ADAMTS-7, A disintegrin and metalloproteinase with thrombospondin motifs 7; COMP, cartilage oligomeric matrix protein.

was downregulated in the decidual tissue of the human subjects with SA compared with the corresponding control group, as demonstrated by immunohistochemistry, western blot and 
A
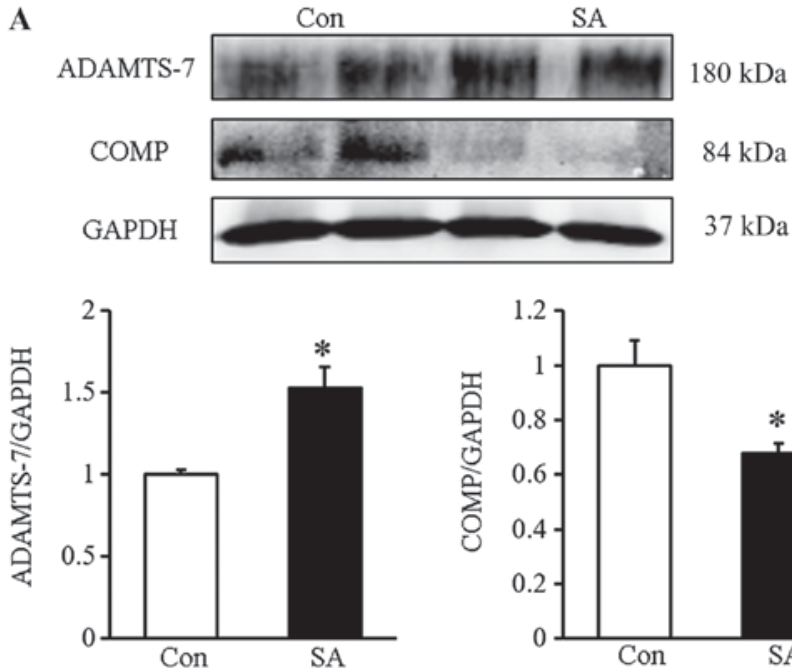

B
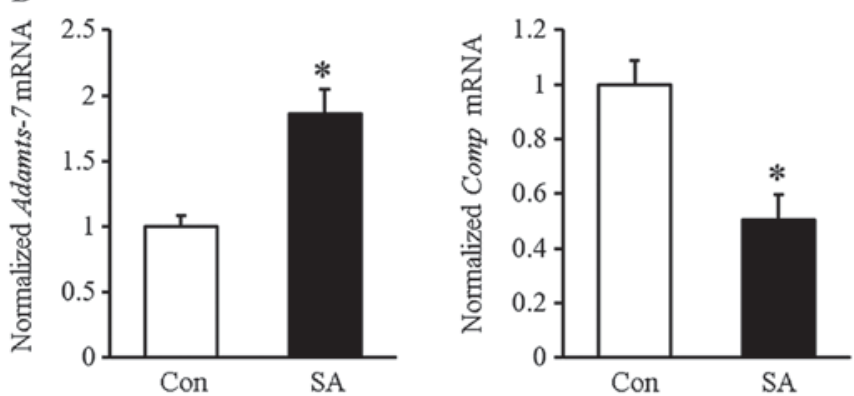

Figure 6. Expression of ADAMTS-7 and COMP in human decidua (A) Protein levels of ADAMTS-7 and COMP in human decidua by western blotting (n=6). (B) mRNA level of Adamts-7 and Comp in human decidua by reverse transcription-quantitative polymerase chain reaction $(n=6) .{ }^{*} \mathrm{P}<0.05$ vs. Con. ADAMTS-7, A disintegrin and metalloproteinase with thrombospondin motifs 7; COMP, cartilage oligomeric matrix protein; Con, control; $\mathrm{SA}$, spontaneous abortion.

RT-qPCR. We also found that ADAMTS-7 was negatively correlated with COMP in human decidual tissue. Thus, in both mice and humans, ADAMTS-7 and its substrate COMP were proposed to play an important role in the pathological process of SA.

The ADAMTS family consists of 19 secreted multidomain proteolytic enzymes and participates in a variety of pathophysiological activities, including extracellular assembly and degradation, hemostasis, organogenesis, angiogenesis, cancer and arthritis (30). ADAMTS-7, a member of the ADAMTS family, is mainly expressed in tendons, ligaments, cartilage and skeletal muscle (31). Our study suggested that ADAMTS-7 could also be detected in the uterus. Previous studies focused on the role of ADAMTS-7 in cartilage degradation, osteoarthritis, collagen-induced arthritis and cardiovascular disease $(10,22,23,32,33)$. The expression of ADAMTS-7 was influenced by a series of factors, including mechanical stimulation, proinflammatory cytokines and oxidative stress (34). The expression of ADAMTS-7 was increased by inflammatory cytokines and decreased by anti-inflammatory cytokines $(23,24)$. It was also reported that ADAMTS-7 and tumor necrosis factor alpha (TNF-a) form a positive feedback loop during the regulation of cartilage degradation and the progression of osteoarthritis $(33,35)$. As demonstrated by previous studies, SA was closely associated with inflammation. Elevated TNF- $\alpha$ was also found to participate in the occurrence of recurrent SA (36). In this study, we found that ADAMTS-7 was increased in the decidual tissues of both mice and humans suffering from SA, which implied that ADAMTS-7 was involved in the pathological process of SA. Moreover, is was reported that subjects suffering from SA were more likely to experience cardiovascular disease later in life. Our findings suggested that ADAMTS-7, which is an important factor involved in cardiovascular disease, also participates in the pathological process of SA. Thus, this study will be helpful toward a better understanding of the etiology of SA.

ADAMTS-7 used to be regarded as a novel locus associated with vascular atherosclerosis by stimulating VSMC migration through the degradation of COMP (24,26,37-41). COMP, an extracellular matrix protein mainly expressed in cartilage and bone tissue $(42,43)$, was also reported as a substrate of ADAMTS-7 and plays a pivotal role in multiple epiphyseal dysplasia and cancer (44-46). To assess whether COMP is associated with ADAMTS-7, the expression of COMP was also determined in the decidual tissues of mice and humans. As demonstrated by immunohistochemistry, COMP was decreased in the LPS-treated group and SA group compared with the corresponding control group. Thus, it was hypothesized that the downregulation of COMP was associated with the upregulation of ADAMTS-7. Spearman's analysis further confirmed our hypothesis and revealed that COMP was negatively correlated with ADAMTS-7. Thus, upregulation of ADAMTS-7 and downregulation of COMP were associated with SA.

However, there are also limitations in this study. First, we only described the expression of ADAMTS-7 and COMP in the decidua of both the LPS-treated mice and human subjects with SA. A functional study of ADAMTS-7 and COMP in SA as well as a determination of the potential regulatory molecular mechanism of ADAMTS-7 and COMP in the pathological process of SA still remain to be performed.

In conclusion, the present study, for the first time, presents the expression levels of ADAMTS-7 and COMP in the decidual tissues of mice and humans suffering from SA. The findings suggest that upregulation of ADAMTS-7 and downregulation of COMP are associated with SA. In addition, a negative correlation was found between ADAMTS-7 and COMP in both mice and humans. Further studies are required to elucidate the potential mechanisms underlying the role of ADAMTS-7 and COMP in SA.

\section{Acknowledgements}

Not applicable.

\section{Funding}

The present study was supported by the Key Project Grant of Natural Science Foundation of Hubei Province (grant no. 2015CFA074).

\section{Availability of data and materials}

The datasets used and/or analyzed during the current study are available from the corresponding author on reasonable request. 


\section{Authors' contributions}

YM and JY were involved in the study design and preparation of the manuscript. YM, DZ and NY carried out the experiments. JD and YM analyzed the data, drafted the manuscript and critically discussed the results with JY.

\section{Ethics approval and consent to participate}

Animal procedures were approved by the Animal Use Committees of Renmin Hospital of Wuhan University (Wuhan, China). The studies involving patients were approved by the Human Research Ethics Committees of Renmin Hospital of Wuhan University (Wuhan, China). Written informed consent was obtained from all participants.

\section{Patient consent for publication}

Not applicable.

\section{Competing interests}

The authors declare that they have no competing interests.

\section{References}

1. Stirrat GM: Recurrent miscarriage. Lancet 336: 673-675, 1990.

2. Jivraj S, Anstie B, Cheong YC, Fairlie FM, Laird SM and Li TC: Obstetric and neonatal outcome in women with a history of recurrent miscarriage: A cohort study. Hum Reprod 16: 102-106, 2001.

3. Hughes GR: Thrombosis, abortion, cerebral disease, and the lupus anticoagulant. Br Med J (Clin Res Ed) 287: 1088-1089, 1983.

4. Geva E, Lerner-Geva L, Burke M, Vardinon N, Lessing JB and Amit A: Undiagnosed systemic lupus erythematosus in a cohort of infertile women. Am J Reprod Immunol 51: 336-340, 2004.

5. Hefler-Frischmuth K, Walch K, Hefler L, Tempfer C and Grimm C: Serologic markers of autoimmunity in women with recurrent pregnancy loss. Am J Reprod Immunol 77: e12635, 2017.

6. Vitagliano A, Noventa M and Gizzo S: Autoimmunity, systemic inflammation, and their correlation with repeated implantation failure and recurrent miscarriage: Is chronic endometritis the missing piece of the jigsaw? Am J Reprod Immunol 77: e12597, 2017.

7. Mekinian A, Cohen J, Alijotas-Reig J, Carbillon L, Nicaise-Roland P, Kayem G, Daraï E, Fain O and Bornes M: Unexplained recurrent miscarriage and recurrent implantation failure: Is there a place for immunomodulation? Am J Reprod Immunol 76: 8-28, 2016.

8. Comba C, Bastu E, Dural O, Yasa C, Keskin G, Ozsurmeli M, Buyru F and Serdaroglu H: Role of inflammatory mediators in patients with recurrent pregnancy loss. Fertil Steril 104: 1467-1474.e1, 2015.

9. Ahmed SK, Mahmood N, Malalla ZH, Alsobyani FM, Al-Kiyumi IS and Almawi WY: C-reactive protein gene variants associated with recurrent pregnancy loss independent of CRP serum levels: A case-control study. Gene 569: 136-140, 2015.

10. Zhang Y, Wei F and Liu CJ: Overexpression of ADAMTS-7 leads to accelerated initiation and progression of collagen-induced arthritis in mice. Mol Cell Biochem 404: 171-179, 2015.

11. Bengtsson E, Hultman K, Dunér P, Asciutto G, Almgren P, Orho-Melander M, Melander O, Nilsson J, Hultgårdh-Nilsson A and Gonçalves I: ADAMTS-7 is associated with a high-risk plaque phenotype in human atherosclerosis. Sci Rep 7: 3753, 2017.

12. Liu CJ: The role of ADAMTS-7 and ADAMTS-12 in the pathogenesis of arthritis. Nat Clin Pract Rheumatol 5: 38-45, 2009.

13. Galgani M, Insabato L, Calì G, Della Gatta AN, Mirra P, Papaccio F, Santopaolo M, Alviggi C, Mollo A, Strina I, et al: Regulatory $\mathrm{T}$ cells, inflammation, and endoplasmic reticulum stress in women with defective endometrial receptivity. Fertil Steril 103: 1579-1586.e1, 2015.
14. Nakashima A, Ito M, Shima T, Bac ND, Hidaka T and Saito S: Accumulation of IL-17-positive cells in decidua of inevitable abortion cases. Am J Reprod Immunol 64: 4-11, 2010.

15. Wang WJ, Hao CF, Yi-Lin, Yin GJ, Bao SH, Qiu LH and Lin QD: Increased prevalence of T helper 17 (Th17) cells in peripheral blood and decidua in unexplained recurrent spontaneous abortion patients. J Reprod Immunol 84: 164-170, 2010.

16. Wang SS, Zhang W, Zhang YQ, Zhao Y, Liu Y, Li JK, Zhang HX, Cheng L and Nie L: IL-17A enhances ADAMTS-7 expression through regulation of TNF- $\alpha$ in human nucleus pulposus cells. J Mol Histol 46: 475-483, 2015.

17. Mosca L, Benjamin EJ, Berra K, Bezanson JL, Dolor RJ, Lloyd-Jones DM, Newby LK, Piña IL, Roger VL, Shaw LJ, et al: Effectiveness-based guidelines for the prevention of cardiovascular disease in women-2011 update: A guideline from the American Heart Association. J Am Coll Cardiol 57: 1404-1423, 2011.

18. Wu P, Haththotuwa R, Kwok CS, Babu A, Kotronias RA, Rushton C, Zaman A, Fryer AA, Kadam U, Chew-Graham CA and Mamas MA: Preeclampsia and future cardiovascular health: A systematic review and meta-analysis. Circ Cardiovasc Qual Outcomes 10: pii: e003497, 2017.

19. Ranthe MF, Andersen EA, Wohlfahrt J, Bundgaard H, Melbye M and Boyd HA: Pregnancy loss and later risk of atherosclerotic disease. Circulation 127: 1775-1782, 2013.

20. Oliver-Williams CT, Heydon EE, Smith GC and Wood AM: Miscarriage and future maternal cardiovascular disease: A systematic review and meta-analysis. Heart 99: 1636-1644, 2013.

21. Wagner MM, Bhattacharya S, Visser J, Hannaford PC and Bloemenkamp KW: Association between miscarriage and cardiovascular disease in a Scottish cohort. Heart 101: 1954-1960, 2015.

22. Wu W, Wang H, Yu C, Li J, Gao Y, Ke Y, Wang Y, Zhou Y and Zheng J: Association of ADAMTS-7 levels with cardiac function in a rat model of acute myocardial infarction. Cell Physiol Biochem 38: 950-958, 2016.

23. Kessler T, Zhang L, Liu Z, Yin X, Huang Y, Wang Y, Fu Y, Mayr M, Ge Q, Xu Q, et al: ADAMTS-7 inhibits re-endothelialization of injured arteries and promotes vascular remodeling through cleavage of thrombospondin-1. Circulation 131: 1191-1201, 2015.

24. Wang L, Zheng J, Bai X, Liu B, Liu CJ, Xu Q, Zhu Y, Wang N, Kong W and Wang X: ADAMTS-7 mediates vascular smooth muscle cell migration and neointima formation in balloon-injured rat arteries. Circ Res 104: 688-698, 2009.

25. Riessen R, Fenchel M, Chen H, Axel DI, Karsch KR and Lawler J: Cartilage oligomeric matrix protein (thrombospondin-5) is expressed by human vascular smooth muscle cells. Arterioscler Thromb Vasc Biol 21: 47-54, 2001.

26. Du Y, Wang Y, Wang L, Liu B, Tian Q, Liu CJ, Zhang T, Xu Q, Zhu Y, Ake O, et al: Cartilage oligomeric matrix protein inhibits vascular smooth muscle calcification by interacting with bone morphogenetic protein-2. Circ Res 108: 917-928, 2011.

27. Bond AR, Hultgårdh-Nilsson A, Knutsson A, Jackson CL and Rauch U: Cartilage oligomeric matrix protein (COMP) in murine brachiocephalic and carotid atherosclerotic lesions. Atherosclerosis 236: 366-372, 2014.

28. Livak KJ and Schmittgen TD: Analysis of relative gene expression data using real-time quantitative PCR and the 2(-Delta Delta C(T)) method. Methods 25: 402-408, 2001.

29. Puhl SL, Kazakov A, Müller A, Fries P, Wagner DR, Böhm M, Maack C and Devaux Y: Adenosine A1 receptor activation attenuates cardiac hypertrophy and fibrosis in response to $\alpha 1$-adrenoceptor stimulation in vivo. Br J Pharmacol 173: 88-102, 2016.

30. Wagstaff L, Kelwick R, Decock J and Edwards DR: The roles of ADAMTS metalloproteinases in tumorigenesis and metastasis. Front Biosci (Landmark Ed) 16: 1861-1872, 2011.

31. Liu CJ, Kong W, Ilalov K, Yu S, Xu K, Prazak L, Fajardo M, Sehgal B and Di Cesare PE: ADAMTS-7: A metalloproteinase that directly binds to and degrades cartilage oligomeric matrix protein. FASEB J 20: 988-990, 2006.

32. GuoF,Lai Y,Tian Q,Lin EA, Kong Land Liu C: Granulin-epithelin precursor binds directly to ADAMTS-7 and ADAMTS-12 and inhibits their degradation of cartilage oligomeric matrix protein. Arthritis Rheum 62: 2023-2036, 2010.

33. Lai Y, Bai X, Zhao Y, Tian Q, Liu B, Lin EA, Chen Y, Lee B, Appleton CT, Beier F, et al: ADAMTS-7 forms a positive feedback loop with TNF- $\alpha$ in the pathogenesis of osteoarthritis. Ann Rheum Dis 73: 1575-1584, 2014. 
34. Jones GC and Riley GP: ADAMTS proteinases: A multi-domain, multi-functional family with roles in extracellular matrix turnover and arthritis. Arthritis Res Ther 7: 160-169, 2005.

35. Buckland J: Osteoarthritis: Positive feedback between ADAMTS-7 and TNF in OA. Nat Rev Rheumatol 9: 566, 2013.

36. Li S, Wang L, Xing Z, Huang Y and Miao Z: Expression level of TNF- $\alpha$ in decidual tissue and peripheral blood of patients with recurrent spontaneous abortion. Cent Eur J Immunol 42 156-160, 2017.

37. Bauer RC, Tohyama J, Cui J, Cheng L, Yang J, Zhang X, Ou K, Paschos GK, Zheng XL, Parmacek MS, et al: Knockout of Adamts7, a novel coronary artery disease locus in humans, reduces atherosclerosis in mice. Circulation 131: 1202-1213, 2015.

38. Patel RS and Ye S: ADAMTS7: A promising new therapeutic target in coronary heart disease. Expert Opin Ther Targets 17: 863-867, 2013.

39. Hanby HA and Zheng XL: Biochemistry and physiological functions of ADAMTS7 metalloprotease. Adv Biochem 1, 2013.

40. You L, Tan L, Liu L, Shen R, Chaugai S, Wang DW and Cui W: ADAMTS7 locus confers high cross-race risk for development of coronary atheromatous plaque. Mol Genet Genomics 291: $121-128,2016$

41. Du Y, Gao C, Liu Z, Wang L, Liu B, He F, Zhang T, Wang Y, Wang $\mathrm{X}, \mathrm{Xu} \mathrm{M}$, et al: Upregulation of a disintegrin and metalloproteinase with thrombospondin motifs- 7 by miR-29 repression mediates vascular smooth muscle calcification. Arterioscler Thromb Vasc Biol 32: 2580-2588, 2012.
42. Müller G, Michel A and Altenburg E: COMP (cartilage oligomeric matrix protein) is synthesized in ligament, tendon, meniscus, and articular cartilage. Connect Tissue Res 39: 233-244, 1998.

43. Tan Kand Lawler J: The interaction of Thrombospondins with extracellular matrix proteins. J Cell Commun Signal 3: 177-187, 2009.

44. Lin WD, Chou IC, Wang CH and Tsai FJ: Novel mutations in the cartilage oligomeric matrix protein gene identified in two Taiwanese patients with pseudoachondroplasia and multiple epiphyseal dysplasia. Pediatr Neonatol: 1-3, 2017.

45. Englund E, Bartoschek M, Reitsma B, Jacobsson L, Escudero-Esparza A, Orimo A, Leandersson K, Hagerling C, Aspberg A, Storm P, et al: Cartilage oligomeric matrix protein contributes to the development and metastasis of breast cancer. Oncogene 35: 5585-5596, 2016.

46. Englund E, Canesin G, Papadakos KS, Vishnu N, Persson E, Reitsma B, Anand A, Jacobsson L, Helczynski L, Mulder H, et al: Cartilage oligomeric matrix protein promotes prostate cancer progression by enhancing invasion and disrupting intracellular calcium homeostasis. Oncotarget 8: 98298-98311, 2017.

(i) () $९$ This work is licensed under a Creative Commons (c) ${ }_{\mathrm{EY}} \mathrm{NG}$ NO Attribution-NonCommercial-NoDerivatives 4.0 International (CC BY-NC-ND 4.0) License. 